\title{
Nine contemporary therapeutic directions in heart failure
}

\author{
Zaid Almarzooq, ${ }^{1}$ Manan Pareek, ${ }^{1,2}$ Lauren Sinnenberg, ${ }^{1}$ Muthiah Vaduganathan, ${ }^{1}$ \\ Mandeep R. Mehra ${ }^{\top}$
}

${ }^{1}$ Brigham and Women's Hospital Heart and Vascular Center and Harvard Medical School, Boston, Massachusetts, USA

${ }^{2}$ Department of Cardiology, North Zealand Hospital, Hillerød Denmark

\section{Correspondence to}

Professor Mandeep R. Mehra, Brigham and Women's Hospital Heart and Vascular Center and Harvard Medical School, Boston MA 02115, USA; mmehra@ bwh.harvard.edu

ZA and MP are joint first authors.

Received 5 February 2019 Revised 8 February 2019 Accepted 11 February 2019
Check for updates

(C) Author(s) (or their employer(s)) 2019. No commercial re-use. See rights and permissions. Published by BMJ.

To cite: Almarzooq $Z_{\text {, }}$

Pareek M, Sinnenberg $L$,

et al. Heart Asia

2019:11:e011150.

doi:10.1136/

heartasia-2018-011150

\section{ABSTRACT}

The global burden of heart failure has continued to increase dramatically with 26 million people affected and an estimated health expenditure of $\$ 31$ billion worldwide. Several practice-influencing studies were reported recently, bringing advances along many frontiers in heart failure, particularly heart failure with reduced ejection fraction. In this article, we discuss nine distinct therapeutic areas that were significantly influenced by this scientific progress. These distinct areas include the emergence of sodium-glucose cotransporter-2 inhibitors, broadening the application of angiotensin-neprilysin inhibition, clinical considerations in therapy withdrawal in those patients with heart failure that 'recover' myocardial function, benefits of low-dose direct oral anticoagulants in sinus rhythm, targeted therapy for treating cardiac amyloidosis, usefulness of mitral valve repair in heart failure, the advent of newer left ventricular assist devices for advanced heart failure, the role of ablation in atrial fibrillation in heart failure, and finally the use of wearable defibrillators to address sudden death.

\section{INTRODUCTION}

The global burden of heart failure has continued to increase dramatically with 26 million people affected and an estimated health expenditure of $\$ 31$ billion worldwide. ${ }^{12}$ Several practice-influencing studies were reported within the past year, bringing advances along many frontiers in heart failure, particularly heart failure with reduced ejection fraction. In this article, we discuss nine therapeutic areas that were significantly influenced by this scientific progress (table 1).

\section{Sodium-glucose cotransporter-2 inhibitors and prevention of cardiovascular outcomes in patients with type 2 diabetes: a case of collateral benefit}

Cardiovascular disease is the leading cause of morbidity and mortality among patients with type 2 diabetes mellitus. ${ }^{3}$ However, despite strong indications of a causal link between hyperglycaemia and microvascular disease, ${ }^{4}$ a direct protective effect of intensive glucose-lowering alone may take up to a decade to demonstrate cardiovascular outcome benefits. Although glucose-lowering drugs were generally expected to improve cardiovascular outcomes, a widely publicised 2007 meta-analysis of 42 randomised trials of the peroxisome proliferator-activated receptor agonist rosiglitazone that suggested a greater risk of myocardial infarction (MI) and cardiovascular mortality with its use dramatically changed this perception. ${ }^{5}$ Subsequently, the US
Food and Drug Administration (FDA) and the European Medicines Agency began requiring comprehensive evaluation of the cardiovascular safety of all new antidiabetic agents. ${ }^{67}$ Drug classes tested under these new requirements include dipeptidyl peptidase-4 inhibitors, glucagon-like peptide 1 receptor agonists and sodium-glucose cotransporter-2 inhibitors (SGLT2i), all of which exert their glucose-lowering effect through distinct mechanisms. SGLT2 inhibition in the proximal tubule of the nephron leads to glucosuria, diuresis, weight loss and blood pressure lowering. ${ }^{8}$ While all subsequently tested medications for type 2 diabetes mellitus have reached the formal non-inferiority criteria, that is, demonstrated safety for a composite cardiovascular endpoint most often comprising cardiovascular death, MI or stroke, the class of SGLT2i also signalled a reduction in composite cardiovascular risk and in the risk of heart failure. ${ }^{9-13}$

Results from the cardiovascular outcome trial of the third SGLT2i, DECLARE-TIMI 58 (Dapagliflozin Effect on Cardiovascular Events-Thrombolysis in Myocardial Infarction 58), were recently reported. ${ }^{14}$ Patients with type 2 diabetes mellitus either with or at risk for atherosclerotic cardiovascular disease were randomised to dapagliflozin $10 \mathrm{mg}$ daily $(\mathrm{n}=8582)$ or placebo $(\mathrm{n}=8578)$ and evaluated for one primary safety endpoint and two coprimary efficacy endpoints. At a median of 4.2 years, dapagliflozin fulfilled the non-inferiority criterion for the primary safety endpoint, a composite of cardiovascular death, MI or ischaemic stroke, but this endpoint did not reach superiority. However, the composite of cardiovascular death or hospitalisation for heart failure, the other coprimary efficacy endpoint, was significantly reduced with dapagliflozin (HR 0.83 , 95\% CI 0.73 to 0.95 ; $\mathrm{p}=0.005$ ) and was driven by a lower risk of hospitalisation for heart failure.

The most impressive risk reductions in all three cardiovascular outcome studies of SGLT2 $\mathrm{i}$ were seen for the heart failure endpoint. This was recently examined in an individual patient-level meta-analysis which reported promising reductions in heart failure in patients both with and without pre-existing heart failure or established atherosclerotic cardiovascular disease, and without significant between-study heterogeneity. ${ }^{15}$ Although the precise mechanisms behind these benefits have not been fully elucidated, it has been suggested that the reduction in hospitalisation for heart failure may be related to the therapy's favourable renal, haemodynamic and metabolic effects. ${ }^{1617}$ Given the therapeutic role in prevention of heart failure events in at-risk populations, this drug class has been incorporated into the 
Table 1 Summary of contemporary therapeutic directions in heart failure

\begin{tabular}{|c|c|c|}
\hline Therapeutic direction & Clinical trial* & Key findings \\
\hline $\begin{array}{l}\text { Sodium-glucose cotransporter-2 inhibition with } \\
\text { dapagliflozin in patients with type } 2 \text { diabetes } \\
\text { mellitus with or at risk for atherosclerotic } \\
\text { cardiovascular disease. }\end{array}$ & DECLARE-TIMI 58 & $\begin{array}{l}\text { The composite of cardiovascular death or } \\
\text { hospitalisation for heart failure was significantly } \\
\text { reduced with dapagliflozin and was driven by a } \\
\text { lower risk of hospitalisation for heart failure. }\end{array}$ \\
\hline $\begin{array}{l}\text { Angiotensin-neprilysin inhibition with sacubitril/ } \\
\text { valsartan in patients hospitalised for acute } \\
\text { decompensated heart failure. }\end{array}$ & PIONEER-HF & $\begin{array}{l}\text { The time-averaged reduction in NT-proBNP was } \\
\text { significantly more reduced with sacubitril/valsartan } \\
\text { compared with enalapril. }\end{array}$ \\
\hline
\end{tabular}

Withdrawal of heart failure medications in patients with presumed recovery of dilated cardiomyopathy.

TRED-HF

Relapse (study-specific criteria that included clinical symptoms of heart failure) was significantly greater in the medication withdrawal group compared with the medication continuation group.

COMMANDER-HF

Anticoagulation with very low-dose rivaroxab in patients with chronic heart failure with reduced ejection fraction, coronary artery disease and sinus rhythm.

Use of the small molecule transthyretin ATTR-ACT tetramer stabiliser, tafamidis, in patients with transthyretin amyloid cardiomyopathy and New York Heart Association class I-III symptoms.

Percutaneous, transcatheter repair using the MitraClip device in patients with heart failure and secondary mitral valve regurgitation.

MITRA-FR

COAPT

MOMENTUM 3

The fully magnetically levitated centrifugal continuous-flow circulatory pump, HeartMate 3 , in patients with advanced heart failure refractory to standard medical therapy.

Catheter ablation for patients with heart failure CASTLE-AF and symptomatic paroxysmal or persistent atrial fibrillation.

Wearable cardioverter-defibrillators in patients VEST with acute myocardial infarction and severe left

ventricular dysfunction.

*Note that we have included only trials published following peer review.

ATTR-ACT, Transthyretin Amyloidosis Cardiomyopathy Clinical Trial; CASTLE-AF, Catheter Ablation vs Standard Conventional Therapy in Patients with Left Ventricular Dysfunction and Atrial Fibrillation; COAPT, Cardiovascular Outcomes Assessment of the MitraClip Percutaneous Therapy for Heart Failure Patients with Functional Mitral Regurgitation ; COMMANDER-HF, Study to Assess the Effectiveness and Safety of Rivaroxaban in Reducing the Risk of Death, Myocardial Infarction, or Stroke in Participants with Heart Failure and Coronary Artery Disease Following an Episode of Decompensated Heart Failure; DECLARE-TIMI 58, Dapagliflozin Effect on Cardiovascular Events-Thrombolysis in Myocardial Infarction 58; MITRA-FR, Percutaneous Repair with the MitraClip Device for Severe Functional/Secondary Mitral Regurgitation; MOMENTUM 3, Multicenter Study of MagLev Technology in Patients Undergoing Mechanical Circulatory Support Therapy with HeartMate 3 ; NT-proBNP, N-terminal pro-B-type natriuretic peptide; PIONEER-HF, the comParlson Of sacubitril/valsartaN vs Enalapril on Effect on nt-pRo-bnp in patients stabilized from an acute Heart Failure episode; TRED-HF, withdrawal of pharmacological therapy for heart failure in recovered dilated cardiomyopathy; VEST, Vest Prevention of Early Sudden Death Trial.

most recent consensus statement by the European Association for the Study of Diabetes and the American Diabetes Association as second-line therapy (after metformin) in patients with type 2 diabetes mellitus with cardiovascular disease. ${ }^{18}$ Ongoing studies are assessing whether these agents may be useful in the treatment of heart failure, including in those who do not have diabetes mellitus (NCT03036124, NCT03619213, NCT03057977, NCT03057951, NCT03521934).

\section{Angiotensin-neprilysin inhibition in acute decompensated heart failure: an opportunity for targeted implementation} Acute decompensated heart failure (ADHF) is an important cause of morbidity and mortality worldwide. ${ }^{19}$ For decades, the standard of care in acute heart failure has been diuretics and haemodynamic support with vasodilators. Prior inpatient studies evaluating the utility of various agents in ADHF have been largely negative. ${ }^{20-22}$ Sacubitril-valsartan is an angiotensin receptor-neprilysin inhibitor that has been approved by the FDA for the treatment of chronic heart failure with reduced ejection fraction. $^{23}$ The landmark PARADIGM-HF (Prospective Comparison of ARNI with ACEI to Determine Impact on Global Mortality and Morbidity in Heart Failure) trial showed improved cardiovascular mortality and heart failure hospitalisation with the use of sacubitril/valsartan compared with enalapril in ambulatory patients with chronic heart failure with reduced ejection fraction. However, patients with ADHF requiring intravenous diuretics were excluded from this trial. ${ }^{24}$

Unfortunately, since the dissemination of the PARADIGM-HF trial results and the regulatory approval of sacubitril/valsartan, its therapeutic uptake in the USA and worldwide has been sluggish. Initiation of this novel class of therapies in patients hospitalised with ADHF may present a unique opportunity to optimise therapy. ${ }^{25}$ The PIONEER-HF (the comParIson Of sacubitril/valsartaN vs Enalapril on Effect on nt-pRo-bnp in

\section{Practice implications}

Incorporated into the most recent international consensus statement as second-line therapy (after metformin) in patients with type 2 diabetes mellitus with cardiovascular disease.

Sacubitril-valsartan may be considered for inpatients as this will simplify the algorithm for inpatient and subsequently outpatient heart failure management.

probatients thought to be in recovery are cautious in withdrawing guideline-directed medical therapy in patients with improved left ventricular function.

There does not appear to be a role for low-dose anticoagulation among patients with heart failure who are in sinus rhythm.

Tafamidis is a safe and effective therapeutic option in patients with early-stage transthyretin amyloid cardiomyopathy.

A proposed cardiomyopathy-dominant form of mitral regurgitation does not appear to benefit from percutaneous transcatheter repair.

A proposed mitral regurgitation-dominant form of myocardial disease on fully supported medical therapy may benefit from percutaneous transcatheter repair.

The HeartMate 3 device is superior in terms of reducing complications associated with left ventricular assist systems.

Catheter ablation is an effective therapeutic option in carefully selected patients with atrial fibrillation and left ventricular dysfunction.

A clear role for wearable cardioverter-defibrillators in this patient population is not yet established. 
patients stabilized from an acute Heart Failure episode) trial was a smaller, randomised clinical trial that sought to assess the safety and efficacy of initiating sacubitril/valsartan among patients hospitalised with ADHF. $^{26}$ Eligible patients $(n=881)$ were randomised to sacubitril/valsartan ( $97 \mathrm{mg}$ of sacubitril with $103 \mathrm{mg}$ of valsartan twice daily) or enalapril (10 mg twice daily). Patients were deemed eligible for inclusion and randomisation if their left ventricular ejection fraction (LVEF) was $\leq 40 \%$ within the past 6 months, had an elevated $\mathrm{N}$-terminal pro-B-type natriuretic peptide (NT-proBNP) $\geq 1600 \mathrm{pg} / \mathrm{mL}$ or B-type natriuretic peptide (BNP) $\geq 400 \mathrm{pg} / \mathrm{mL}$ during the current hospitalisation, and met the definition of stable status that included no increase in intravenous diuretic dose within the last 6 hours and no intravenous inotropic drugs within the last 24 hours. Importantly, $36 \%$ of patients included in the study were black and $34 \%$ of patients had no history of known heart failure. After 8 weeks of follow-up, the investigators showed that sacubitril-valsartan reduced the primary outcome, that is, time-averaged reduction in NT-proBNP, to a greater degree than enalapril $(-46.7 \%$ vs $-25.3 \%$, HR $0.71,95 \%$ CI 0.63 to $0.81 ; \mathrm{p}<0.001)$. In addition, sacubitril/valsartan also reduced the secondary composite outcome of death, rehospitalisation for heart failure, transition to left ventricular assist device or listing for cardiac transplant. This should be interpreted with caution as the study was not sufficiently powered to adequately assess clinical outcomes. Notably, almost half of the patients had no prior exposure to a renin-angiotensin system inhibitor. Reassuringly, there was no significant difference in adverse outcomes that include worsening renal function, hyperkalaemia, hypotension and angioedema.

This study provides data to support initiation of sacubitril/ valsartan for inpatients, even directly before a trial of another renin-angiotensin system inhibitor. This will simplify the algorithm for inpatient and subsequently outpatient heart failure management, and potentially improve therapeutic use of sacubitril/valsartan in practice. Importantly, sacubitril/valsartan is now being evaluated for separate indications in patients with heart failure with preserved ejection fraction (NCT01920711) and postmyocardial left ventricular dysfunction (NCT02924727).

\section{Withdrawal of heart failure-directed therapy in patients with recovered dilated cardiomyopathy: when less is not more}

Multiple longitudinal studies have shown improvements in ventricular function and clinical outcomes in patients with an initial diagnosis of heart failure with reduced ejection fraction. ${ }^{27}$ It remains unclear how this improvement occurs, that is, whether it is due to treatment of the insulting factor or a response to medical therapy. More importantly, this raises the question of whether this is true recovery or merely remission. This further translates into a more clinically salient issue of whether patients with improved ejection fraction still require ongoing medical treatment.

The TRED-HF (withdrawal of pharmacological therapy for heart failure in recovered dilated cardiomyopathy) trial was an open-label, randomised study of withdrawal of guideline-directed medical therapy in 51 patients with heart failure with reduced ejection fraction caused by dilated cardiomyopathy with complete restoration of LVEF ( $>50 \%$ ), left ventricular end diastolic volume index, NT-proBNP $<250 \mathrm{ng} / \mathrm{L}$ and New York Heart Association class I symptoms. ${ }^{28}$ The primary endpoint was relapse within 6 months, defined as one of the following: reduction in LVEF $>10 \%$ and to $<50 \%$, an increase in left ventricular end diastolic volume $>10 \%$ or a twofold rise in NT-proBNP to $>400 \mathrm{ng} / \mathrm{L}$, or clinical symptoms of heart failure. Using a 16-week medication withdrawal protocol and after a follow-up period of 6 months, the study showed a $44 \%$ rate of relapse in the withdrawal arm as compared with none in the continuation arm (Kaplan-Meier estimate of event rate 45.7\%, 95\% CI 28.5 to $67.2 ; \mathrm{p}<0.001)$. After 6 months, patients in the continuation arm crossed over to the discontinuation arm, with relapse occurring in $36 \%$ of patients during the 6 -month follow-up period (Kaplan-Meier estimate of event rate 36.0\%, 95\% CI 20.6 to 57.8). Among the patients who relapsed, all were asymptomatic, and $85 \%$ still had an LVEF $>50 \%$. No deaths were reported in either arm.

Despite this study's small size and single-centre unblinded design, it has important implications on our current practice. The improvement in LVEF seen in patients with dilated cardiomyopathy after initiation of standard medical therapy likely represents remission rather than sustained recovery. However, questions have been raised about the patients included in the study since a significant proportion $(\sim 40 \%)$ had late gadolinium enhancement on cardiac MRI. This study provides further evidence that the majority of patients thought to be in recovery are in fact in remission. The study was not powered to assess for predictors of relapse; however, there were signals for some predictors including advanced age, prescription of a Mineralocorticoid Receptor Antagonist (MRA) before withdrawal, prescription of more than two heart failure medications, increased NT-proBNP concentration and decreased peak global radial strain. Currently, clinicians should be cautious in withdrawing guideline-directed medical therapy in patients with improved or recovered LVEF and, if necessary, careful monitoring is needed. Future studies will need to focus on parameters and biomarkers that can identify patients with a high chance of sustained recovery.

\section{Rivaroxaban in patients with heart failure, sinus rhythm and coronary disease: still no benefit of (low-dose) anticoagulation}

Recent work has suggested the possibility of a link between thrombin-driven pathways and worsening heart failure, raising the question of whether targeting these pathways with anticoagulants could potentially improve clinical outcomes. ${ }^{29}$ Prospective clinical trials including WASH (The Warfarin/Aspirin Study in Heart failure), HELAS (Heart failure Long-term Antithrombotic Study) and WARCEF (The Warfarin vs Aspirin in Reduced Cardiac Ejection Fraction) demonstrated that warfarin did not improve mortality in patients with chronic heart failure. ${ }^{30-32}$ Compared with warfarin, rivaroxaban more directly reduces thrombin generation, and therefore may have a stronger effect on pathways associated with heart failure. In fact, a retrospective analysis of the ATLAS ACS 2-TIMI-51 (Anti-Xa Therapy to Lower Cardiovascular Events in Addition to Standard Therapy in Subjects with Acute Coronary Syndrome-Thrombolysis in Myocardial Infarction 46) trial evaluating rivaroxaban in patients with coronary artery disease suggested the subgroup of patients with heart failure may have derived greater benefit from this therapy compared with others in the study. ${ }^{33}$

The COMMANDER-HF (Study to Assess the Effectiveness and Safety of Rivaroxaban in Reducing the Risk of Death, Myocardial Infarction, or Stroke in Participants with Heart Failure and Coronary Artery Disease Following an Episode of Decompensated Heart Failure) trial assessed whether low-dose rivaroxaban (2.5 $\mathrm{mg}$ twice daily) improves outcomes in patients with chronic heart failure with reduced ejection fraction (LVEF $\leq 45 \%)$ and coronary artery disease in sinus rhythm compared with standard therapy. ${ }^{34}$ Patients were deemed eligible for inclusion and randomisation if they had chronic heart failure $(\geq 3$ months in duration) with an acute worsening in the last 21 days, 
LVEF $\leq 45 \%$, coronary artery disease, in sinus rhythm and with elevated natriuretic peptides (BNP > 200 or NT-proBNP $>800$ ). No significant difference was seen in the composite primary endpoint of death from any cause, MI or stroke among patients randomised to rivaroxaban or placebo $(25.0 \%$ vs $26.2 \%$, HR $0.94,95 \%$ CI 0.84 to $1.05 ; \mathrm{p}=0.27)$. There was also no significant difference in all-cause mortality $(21.8 \%$ vs $22.1 \%$, HR $0.98,95 \%$ CI 0.87 to 1.10 ) between study arms.

Overall, this study does not support the use of very low-dose rivaroxaban in patients with heart failure with reduced ejection fraction and coronary artery disease who are in sinus rhythm. This is likely because thrombin-mediated events are not the main driver of cardiovascular events in patients with recent heart failure hospitalisation, and these patients face strong competing risks of morbidity and mortality related to progression of heart failure. It is unclear whether a higher dose of rivaroxaban or select subgroups of patients with heart failure may have shown a benefit and therefore should be investigated.

\section{Tafamidis in patients with transthyretin amyloid cardiomyopathy: towards targeted therapy}

Transthyretin, also referred to as prealbumin, is primarily synthesised in the liver and forms a homotetramer to transport thyroxine and retinol-binding protein-retinol (vitamin A) complex. ${ }^{35}$ The native transthyretin homotetramer can dissociate into monomers that can misfold and ultimately form amyloid fibrils. Amyloid-related complications occur due to aggregation of the wild-type transthyretin protein over time, also known previously as senile systemic amyloidosis, or due to various mutations of the transthyretin gene that are associated with an increased rate of tetramer dissociation, the rate-limiting step in amyloidogenesis. Two phenotypic presentations of the disease predominate: transthyretin familial amyloid polyneuropathy (TTR-FAP) and transthyretin cardiomyopathy (TTR-CM). TTR-CM is a life-threatening disease caused by intramyocardial deposition of transthyretin-derived amyloid fibrils. This leads to progressive ventricular wall thickening and stiffness of the heart. Once diagnosed, TTR-CM is associated with a life expectancy of 2-6 years. ${ }^{36}$ Multiple approaches have been proposed for the treatment of TTR-CM. These include reducing or halting mutant TTR production in the liver or stabilising the TTR tetramer to prevent dissociation into amyloidogenic monomers. Multiple trials have assessed these various approaches with regard to halting the progression of neuropathy related to TTR-FAP. ${ }^{37-39}$ The ATTR-ACT (Transthyretin Amyloidosis Cardiomyopathy Clinical Trial) was designed to evaluate the efficacy and safety of the small molecule TTR tetramer stabiliser, tafamidis, in patients with TTR-CM and New York Heart Association class I-III. ${ }^{40}$

In ATTR-ACT, 441 patients from 13 countries with biopsy-proven TTR-CM were randomised in 2:1:2 ratio to tafamidis $80 \mathrm{mg}$ daily, tafamidis $20 \mathrm{mg}$ daily or placebo. ${ }^{41}$ After 30 months of follow-up, tafamidis was associated with a significant reduction in the primary composite outcome of all-cause mortality and cardiovascular hospitalisation (win ratio $1.70,95 \%$ CI 1.26 to $2.29 ; \mathrm{p}<0.001$ ). Tafamidis also demonstrated reductions in both all-cause mortality $(29.5 \%$ vs $42.9 \%$, HR $0.70,95 \%$ CI 0.51 to 0.96 ) and frequency of cardiovascular hospitalisations ( 0.48 vs 0.70 per year; relative risk ratio $0.68,95 \%$ CI 0.56 to $0.81)$. The Kaplan-Meier survival analysis demonstrated a significant reduction in all-cause mortality with the curves diverging after approximately 18 months of treatment. Importantly, this benefit was not seen in patients with New York Heart Association class III symptoms at baseline, suggesting that therapy may need to be initiated early in the course of the disease. Tafamidis was also associated with a significant reduction in secondary outcomes that included a lower rate of decline in the 6 min walk test and led to improved health-related quality of life $(p<0.001$ for each). Safety analysis demonstrated a lower rate of discontinuation of tafamidis due to treatment-related adverse events.

The ATTR-ACT trial is the largest multicentre study of a treatment for TTR-CM. It provides evidence for an effective therapeutic option for patients with early-stage TTR-CM. Future studies will assess the efficacy of reducing transthyretin production in TTR-CM using TTR-specific oligonucleotides (patisiran, Alnylam Pharmaceuticals; inotersen, Ionis Pharmaceuticals) that inhibit the translation of TTR messenger RNA and have been recently FDA-approved for hereditary ATTR amyloidosis with polyneuropathy. ${ }^{37} 3942$

\section{Percutaneous mitral valve repair in patients with secondary mitral regurgitation: controversy or opportunity?}

Contemporary European and North American guidelines recommend surgical treatment in most patients with severe primary chronic mitral regurgitation, with valve repair preferred over replacement. ${ }^{43} 44$ On the other hand, the best treatment for chronic mitral regurgitation that is secondary to severe left ventricular dysfunction, whether ischaemic or non-ischaemic, is controversial. Although the presence of mitral regurgitation is an adverse prognostic marker, ${ }^{45}$ it is unclear whether correction improves clinical outcomes in these individuals as compared with guideline-recommended medical therapy and cardiac resynchronisation therapy.

In 2018, two randomised clinical trials assessing the utility of percutaneous, transcatheter repair with the MitraClip device among patients with heart failure and secondary mitral valve regurgitation were published. In the MITRA-FR trial (Percutaneous Repair with the MitraClip Device for Severe Functional/ Secondary Mitral Regurgitation), patients with severe mitral regurgitation, an LVEF 15\%-40\% and symptoms of heart failure were randomised to either intervention $(n=152)$ or medical treatment only $(n=152) .{ }^{46}$ At 12 months, the primary composite efficacy endpoint of death from any cause or hospitalisation for heart failure had occurred in 83 patients assigned to intervention and 78 patients assigned to medical treatment (OR 1.16, 95\% CI 0.73 to $1.84 ; p=0.53$ ), with no between-group differences in the incidence of its individual components. The COAPT (Cardiovascular Outcomes Assessment of the MitraClip Percutaneous Therapy for Heart Failure Patients with Functional Mitral Regurgitation) investigators randomised patients with moderate-to-severe mitral regurgitation, an LVEF 20\%-50\% and symptoms of heart failure to device versus control. ${ }^{47}$ The primary endpoint, hospitalisation for heart failure, was significantly reduced in the MitraClip arm (24-month event rates 36\% vs 68\% per patientyear, HR $0.53,95 \%$ CI 0.40 to $0.70 ; p<0.001)$. The study also met its primary safety endpoint of freedom from device-related complications at 12 months (97\%, 95\% lower confidence boundary $95 \%$; $<<0.001$ vs the prespecified performance goal of $88 \%$ ). Finally, the $24-$ month rate of death from any cause (29\% vs $46 \%$, HR 0.62 , 95\% CI 0.46 to $0.82 ; \mathrm{p}<0.001$ ) was significantly reduced with intervention.

The diverging results from the two studies add to the controversy of the optimal strategy in this patient population. However, more patients in COAPT than in MITRA-FR may have had truly refractory mitral regurgitation. ${ }^{48}$ For example, in COAPT, patients were to receive optimal medical therapy for heart failure before randomisation and only those in whom the degree of mitral regurgitation did not decrease during the run-in phase were included. The degree of mitral regurgitation 
also appeared to be more severe in COAPT and more clips were used per patient on average. Finally, it has been suggested that there may be two distinct phenotypes of secondary mitral regurgitation, that is, a cardiomyopathy-dominant form that may not benefit from device therapy and a mitral regurgitation-dominant form of myocardial disease on fully supported medical therapy that appeared to be the target of COAPT. Further analyses of these data, particularly of pharmacological regimens before randomisation, are warranted before clear recommendations can be made with respect to who will derive the most benefit from invasive treatment of secondary mitral regurgitation. In addition, the Reshape-HF2 (RandomizEd Study of tHe MitrACliP DEvice in Heart Failure Patients With Clinically Significant Functional Mitral Regurgitation) is ongoing and expected to report its results in 2021 (NCT02444338).

\section{The fully magnetically levitated centrifugal continuous- flow circulatory pump: left ventricular assist systems gain momentum}

Given the increasing burden of patients with advanced heart failure, the use of left ventricular assist systems that provide mechanical circulatory support has also increased rapidly. ${ }^{49}$ Established indications for durable left ventricular assist systems include bridge-to-transplant and destination therapy (lifetime use of devices in transplant ineligible patients). Although early devices improved prognosis, there was a high risk of adverse events, including pump malfunction due to pump thrombosis. ${ }^{50}$ The fully magnetically levitated centrifugal continuous-flow circulatory pump, HeartMate 3, was designed to reduce shear stress on blood elements and subsequently reduce the risk of pump thrombosis. ${ }^{51}$

In the non-blinded, randomised MOMENTUM 3 trial (Multicenter Study of MagLev Technology in Patients Undergoing Mechanical Circulatory Support Therapy with HeartMate 3 ), patients with advanced heart failure refractory to standard medical therapy were assigned to either the centrifugal-flow pump HeartMate 3 or the axial-flow pump HeartMate II. The primary composite endpoint of survival free from disabling stroke or from reoperation to replace or remove a malfunctioning device was first assessed at 6 months in the initially enrolled 294 patients. At this point, significantly more patients in the centrifugal-flow pump group than in the axial-flow pump group had achieved the primary endpoint, establishing both non-inferiority and superiority, a finding driven by the impact of the HeartMate 3 pump on ameliorating pump thrombosis. ${ }^{51}$ In an extended population of 366 patients followed to 2 years, the primary endpoint occurred in 151 of 190 patients in the centrifugal-flow pump group and 106 of 176 patients in the axial-flow pump group (absolute difference, 19\%, 95\% lower confidence boundary $10 \% ; \mathrm{p}<0.001$ for non-inferiority; HR $0.46,95 \% \mathrm{CI}$ 0.31 to $0.69 ; \mathrm{p}<0.001$ for superiority). Although this difference was driven by pump reoperation rates, the overall rate of stroke was also significantly lower among patients assigned to the centrifugal-flow pump. ${ }^{52}$ Furthermore, the use of HeartMate 3 appeared to be cost-effective. ${ }^{53}$

The full trial cohort of MOMENTUM $3(n=1028)$ has now been analyzed and will be reported in March 2019. The device seems promising in terms of reducing complications, and while the risk of de novo pump thrombosis may have been resolved, thrombosis due to ingestion into the pump may still occur. Furthermore, reports of twisting of the outflow graft have been noted, and a surgical clip to secure the pump outflow to the housing has been introduced. ${ }^{54}$ Therefore, although the
HeartMate 3 is certainly a great leap forward, further development in this area is expected.

\section{Catheter ablation for atrial fibrillation with heart failure: the rise of the Castle}

Patients with heart failure often have concomitant atrial fibrillation which is associated with a higher risk of stroke, heart failure hospitalisations and death. ${ }^{55}$ Earlier studies have demonstrated the efficacy of catheter ablation for symptomatic paroxysmal and persistent atrial fibrillation resistant to antiarrhythmic drugs as well as its safety as first-line therapy in these individuals. ${ }^{5657}$ However, high-quality evidence for the optimal management of atrial fibrillation in patients with heart failure with reduced ejection fraction has not been available.

The CASTLE-AF (Catheter Ablation vs Standard Conventional Therapy in Patients with Left Ventricular Dysfunction and Atrial Fibrillation) study was a multicentre, open-label, controlled trial that randomised patients with heart failure and symptomatic paroxysmal or persistent atrial fibrillation to catheter ablation $(n=179)$ or standard medical therapy $(n=184) .{ }^{58}$ Patients were eligible for inclusion and randomisation if they had a documented atrial fibrillation episode within the last 3 months prior to enrolment, LVEF $\leq 35 \%$ measured within the last 6 weeks, New York Heart Association class II-IV symptoms, and failed antiarrhythmic medical therapy or unwillingness to take antiarrhythmic medications. Furthermore, all patients were required to have had an implantable defibrillator or a cardiac resynchronisation therapy defibrillator with remote monitoring abilities implanted. After a median follow-up of 37.8 months, catheter ablation was associated with a significant reduction in the primary outcome that included death from any cause or hospitalisation for heart failure (29\% vs 36\%, HR 0.62, 95\% CI 0.43 to $0.87 ; p=0.007$ ), with both individual endpoints being significantly reduced in the intervention group. In the secondary analyses, catheter ablation was also associated with a significant reduction in cardiovascular death (HR 0.49 , 95\% CI 0.29 to $0.84 ; \mathrm{p}=0.009$ ) and any hospitalisation (HR 0.72, 95\% CI 0.52 to $0.99 ; \mathrm{p}=0.04)$.

CASTLE-AF demonstrated the benefit of catheter ablation in patients with atrial fibrillation and left ventricular dysfunction. However, it is worth noting that this study is limited in its generalisability largely given the highly selected patient population and open-label design. The CABANA (Catheter ABlation vs ANtiarrhythmic Drug Therapy in Atrial Fibrillation) trial, presented at the Heart Rhythm Society Annual Scientific Session, included a much broader population with atrial fibrillation. Despite not showing a significant benefit in the primary composite outcome of all-cause mortality, disabling stroke, cardiac arrest or serious bleeding, their subgroup analysis showed a significant benefit in patients with heart failure (New York Heart Association class $\geq \mathrm{II}$ ). However, we await its formal publication to better understand the results. ${ }^{59}$

\section{Wearable cardioverter-defibrillator after MI: removing the vest}

MIs that result in low LVEF are associated with high rates of sudden cardiac death. ${ }^{60}$ Prior studies have shown a mortality benefit of implantable cardioverter-defibrillators placed months after MI in patients with a reduced ejection fraction. ${ }^{61-63}$ However, studies that assessed the benefits of early implantable cardioverter-defibrillator implantation after MI did not show a reduction in long-term mortality. ${ }^{64} 65$

VEST (Vest Prevention of Early Sudden Death Trial) was a multicentre, randomised, open-label controlled trial that randomised, 
in a 2:1 fashion, patients hospitalised for an acute MI and severe left ventricular dysfunction to a wearable cardioverter-defibrillator $(n=1524)$ or standard medical therapy $(n=778) .{ }^{66}$ Patients were deemed eligible for inclusion and randomisation if they had LVEF $\leq 35 \%$ that was determined $\geq 8$ hours (in those who were medically managed for their MI or had a percutaneous coronary intervention) or $\geq 48$ hours if they underwent coronary artery bypass graft surgery. At 3 months, there was no significant reduction in the primary composite endpoint of sudden death or death from ventricular tachyarrhythmia $(1.6 \%$ vs $2.4 \%$, HR $0.67,95 \%$ CI 0.37 to $1.21 ; p=0.18$ ). However, there was a significant reduction in the secondary outcome of death of any cause (HR $0.64,95 \%$ CI 0.43 to $0.98 ; \mathrm{p}=0.04$ ).

In this study, the use of wearable cardioverter-defibrillators was not associated with a reduction in sudden cardiac deaths up to 90 days in patients with severe left ventricular dysfunction postinfarction. In light of this non-significant finding of the primary endpoint, the finding of a decrease in all-cause mortality should be interpreted with caution. A finding such as this on a secondary endpoint that is not sufficiently powered to demonstrate a difference or corrected for multiplicity should only be considered exploratory. Factors likely to have diminished the measured effect of these devices include the high cross-over rate (19\%) and compliance issues. Awareness, attention and adherence to other guideline-directed medical therapies may have been greater in patients randomised to wearable cardioverter-defibrillators. Future studies will likely investigate the individual determinants of benefit from this intervention so that the population most likely to benefit can be selected.

\section{A spotlight on heart failure in the Asia-Pacific region}

Despite the progress in identifying and understanding appropriate interventions for the global heart failure population, limited data are available mapping the epidemiology, therapeutic needs and effectiveness of therapies in patients in the Asia-Pacific region. ${ }^{67}$ Given marked global differences in biological features (aetiologies, comorbid diseases), social determinants of health (medical costs and healthcare access) and culture (diet, health attitudes), dedicated study of patients in the Asia-Pacific region represents a major unmet need. It is reassuring that contemporary clinical trials are enrolling an increasing proportion of patients from this region; for instance, $28 \%$ of patients in the large ATMOSPHERE (Aliskiren Trial to Minimize Outcomes in Patients with Heart Failure) trial were enrolled from the Asia-Pacific region. Dedicated efforts, including the ASIAN-HF (Asian Sudden Cardiac Death in Heart Failure) registry (NCT01633398), are also defining the heart failure epidemiology of this region. ${ }^{68}$

\section{CONCLUSION}

Some of these clinical trials have challenged our current understandings, while others have brought forth new therapeutic options for particular subsets of our patients. These studies have enriched the discussions and debates at some of our largest cardiovascular scientific meetings. This article summarises the multitude of recent studies conducted in patients who have or are at high risk for developing heart failure. The field is rapidly developing, and we enthusiastically await follow-up studies, real-world data and updated guidelines to assess their impact on daily clinical practice. Given the worldwide burden of heart failure, focused attention in ongoing epidemiological studies and randomised clinical trials is needed in enrolling global populations, including patients living in the Asia-Pacific region.
Contributors This work in review represents a collective work of all the authors. ZA and MP contributed equally. All authors were involved in drafting, editing and intellectual content of this work. The corresponding author was responsible for the idea, format and overall supervision, in addition to editing and writing of key portions of the manuscript.

Funding The authors have not declared a specific grant for this research from any funding agency in the public, commercial or not-for-profit sectors.

Competing interests MV is supported by the KL2/Catalyst Medical Research Investigator Training award from Harvard Catalyst (NIH/NCATS Award UL 1TR002541), and serves on the advisory boards for AstraZeneca, Bayer AG and Baxter Healthcare. MP serves on an advisory board for AstraZeneca and has received speaker honoraria from AstraZeneca, Bayer and Boehringer Ingelheim. MM is a consultant for Abbott, Medtronic, Janssen (a division of Johnson and Johnson), Mesoblast, NupulseCV, Bayer, FineHeart, Xogenex and Portola.

Patient consent for publication Not required.

Provenance and peer review Commissioned; internally peer reviewed.

\section{REFERENCES}

1. Ponikowski P, Anker SD, AlHabib KF, et al. Heart failure: preventing disease and death worldwide. ESC Heart Fail 2014;1:4-25.

2. Benjamin EJ, Muntner P, Alonso A, et al. Heart disease and stroke statistics - 2019 update: a report from the American Heart Association. Circulation 2019;47.

3. Gregg EW, Gu Q, Cheng YJ, et al. Mortality trends in men and women with diabetes, 1971 to 2000. Ann Intern Med 2007;147:149-55.

4. Ross S, Gerstein HC, Eikelboom J, et al. Mendelian randomization analysis supports the causal role of dysglycaemia and diabetes in the risk of coronary artery disease. Eur Heart J 2015:36:1454-62.

5. Nissen SE, Wolski K. Effect of rosiglitazone on the risk of myocardial infarction and death from cardiovascular causes. N Engl J Med 2007;356:2457-71.

6. European Medicines Agency (Committee for Medicinal Products for Human Use). Guideline on clinical investigation of medicinal products in the treatment or prevention of diabetes mellitus [Internet]. Guideline on clinical investigation of medicinal products in the treatment or prevention of diabetes mellitus, 2012. Available: https://www.ema.europa.eu/en/clinical-investigation-medicinal-productstreatment-prevention-diabetes-mellitus [Accessed 31 Dec 2018].

7. Food and Drug Administration. Guidance for Industry on Diabetes Mellitus-Evaluating Cardiovascular Risk in New Antidiabetic Therapies to Treat Type 2 Diabetes [Internet]. Guidance for Industry on Diabetes Mellitus-Evaluating Cardiovascular Risk in New Antidiabetic Therapies to Treat Type 2 Diabetes, 2008. Available: https://www. federalregister.gov/documents/2008/12/19/E8-30086/guidance-for-industry-ondiabetes-mellitus-evaluating-cardiovascular-risk-in-new-antidiabetic [Accessed 1 Jan 2019].

8. Sattar N, Petrie MC, Zinman B, et al. Novel diabetes drugs and the cardiovascular specialist. J Am Coll Cardiol 2017;69:2646-56.

9. Zinman B, Wanner C, Lachin JM, et al. Empagliflozin, cardiovascular outcomes, and mortality in type 2 diabetes. N Engl J Med 2015;373:2117-28.

10. Fitchett $D$, Zinman $B$, Wanner $C$, et al. Heart failure outcomes with empagliflozin in patients with type 2 diabetes at high cardiovascular risk: results of the EMPA-REG OUTCOME ${ }^{\circledR}$ trial. Eur Heart J 2016:37:1526-34.

11. Fitchett $D$, Butler J, van de Borne $P$, et al. Effects of empagliflozin on risk for cardiovascular death and heart failure hospitalization across the spectrum of heart failure risk in the EMPA-REG OUTCOME® trial. Eur Heart J 2018;39:363-70.

12. Neal B, Perkovic V, Mahaffey KW, et al. Canagliflozin and cardiovascular and renal events in type 2 diabetes. N Engl J Med 2017;377:644-57.

13. Rådholm K, Figtree G, Perkovic V, et al. Canagliflozin and heart failure in type 2 diabetes mellitus. Circulation 2018;138:458-68.

14. Wiviott SD, Raz I, Bonaca MP, et al. Dapagliflozin and cardiovascular outcomes in type 2 diabetes. N Eng/ J Med 2019;380:347-57.

15. Zelniker TA, Wiviott SD, Raz I, et al. SGLT2 inhibitors for primary and secondary prevention of cardiovascular and renal outcomes in type 2 diabetes: a systematic review and meta-analysis of cardiovascular outcome trials. The Lancet 2019;393:31-9.

16. Cherney DZI, Perkins BA, Soleymanlou N, et al. Renal hemodynamic effect of sodium-glucose cotransporter 2 inhibition in patients with type 1 diabetes mellitus. Circulation 2014;129:587-97.

17. Heerspink HJL, Perkins BA, Fitchett DH, et al. Sodium glucose cotransporter 2 inhibitors in the treatment of diabetes mellitus: cardiovascular and kidney effects, potential mechanisms, and clinical applications. Circulation 2016;134:752-72.

18. Davies MJ, D'Alessio DA, Fradkin J, et al. Management of hyperglycaemia in type 2 diabetes, 2018. A consensus report by the American diabetes Association (ADA) and the European association for the study of diabetes (EASD). Diabetologia 2018;61:2461-98.

19. Ambrosy AP, Fonarow GC, Butler J, et al. The global health and economic burden of hospitalizations for heart failure: lessons learned from hospitalized heart failure registries. J Am Coll Cardiol 2014;63:1123-33. 
20. Kelly JP, Mentz RJ, Hasselblad V, et al. Worsening heart failure during hospitalization for acute heart failure: insights from the acute study of clinical effectiveness of nesiritide in decompensated heart failure (ASCEND-HF). Am Heart J 2015; 170:298-305.

21. Packer M, O'Connor C, McMurray JJV, et al. Effect of ularitide on cardiovascular mortality in acute heart failure. N Engl J Med 2017;376:1956-64.

22. Konstam MA, Gheorghiade M, Burnett JC, et al. Effects of oral tolvaptan in patients hospitalized for worsening heart failure: the Everest outcome trial. JAMA 2007;297:1319-31.

23. Fala L, Entresto FL. Entresto (Sacubitril/Valsartan): first-in-class angiotensin receptor neprilysin inhibitor FDA approved for patients with heart failure. Am Health Drug Benefits 2015;8:330-4.

24. McMurray JJV, Packer M, Desai AS, et al. Angiotensin-Neprilysin inhibition versus enalapril in heart failure. N Engl J Med 2014;371:993-1004.

25. Bhagat AA, Greene SJ, Vaduganathan $M$, et al. Initiation, continuation, switching, and withdrawal of heart failure medical therapies during hospitalization. JACC Heart Fail 2019;7:1-12.

26. Velazquez EJ, Morrow DA, DeVore AD, et al. Angiotensin-Neprilysin inhibition in acute decompensated heart failure. N Engl J Med 2019;380.

27. Punnoose LR, Givertz MM, Lewis EF, et al. Heart failure with recovered ejection fraction: a distinct clinical entity. J Card Fail 2011;17:527-32.

28. Halliday BP, Wassall R, Lota AS, et al. Withdrawal of pharmacological treatment for heart failure in patients with recovered dilated cardiomyopathy (TRED-HF): an openlabel, pilot, randomised trial. Lancet 2019;393:61-73.

29. Zannad F, Stough WG, Regnault V, et al. Is thrombosis a contributor to heart failure pathophysiology? Possible mechanisms, therapeutic opportunities, and clinical investigation challenges. Int J Cardiol 2013;167:1772-82.

30. Cokkinos DV, Toutouzas PK. Antithrombotic therapy in heart failure: a randomized comparison of warfarin vs. aspirin (HELAS). Eur J Heart Fail 1999;1:419-23.

31. Cleland JGF, Findlay I, Jafri S, et al. The Warfarin/Aspirin study in heart failure (wash): a randomized trial comparing antithrombotic strategies for patients with heart failure. Am Heart J 2004;148:157-64.

32. Homma S, Thompson JLP, Pullicino PM, et al. Warfarin and aspirin in patients with heart failure and sinus rhythm. N Engl J Med 2012;366:1859-69.

33. Korjian S, Braunwald E, Daaboul Y, et al. Usefulness of rivaroxaban for secondary prevention of acute coronary syndrome in patients with history of congestive heart failure (from the ATLAS-ACS-2 TIMI-51 trial). Am J Cardiol 2018;122:1896-901.

34. Zannad F, Anker SD, Byra WM, et al. Rivaroxaban in patients with heart failure, sinus rhythm, and coronary disease. N Engl J Med 2018;379:1332-42.

35. Rapezzi C, Quarta CC, Riva L, et al. Transthyretin-related amyloidoses and the heart: a clinical overview. Nat Rev Cardiol 2010;7:398-408.

36. Pinney JH, Whelan CJ, Petrie A, et al. Senile systemic amyloidosis: clinical features at presentation and outcome. J Am Heart Assoc 2013;2:e000098.

37. Benson MD, Waddington-Cruz M, Berk JL, et al. Inotersen treatment for patients with hereditary transthyretin amyloidosis. N Engl J Med 2018;379:22-31.

38. Coelho T, Maia LF, Martins da Silva A, et al. Tafamidis for transthyretin familial amyloid polyneuropathy: a randomized, controlled trial. Neurology 2012;79:785-92.

39. Adams D, Gonzalez-Duarte A, O'Riordan WD, et al. Patisiran, an RNAi therapeutic, for hereditary transthyretin amyloidosis. N Engl J Med 2018;379:11-21.

40. Maurer MS, Elliott P, Merlini G, et al. Design and rationale of the phase 3 ATTR-ACT clinical trial (Tafamidis in transthyretin cardiomyopathy clinical trial). Circ Heart Fail 2017:10.

41. Maurer MS, Schwartz JH, Gundapaneni B, et al. Tafamidis treatment for patients with transthyretin amyloid cardiomyopathy. N Eng/ J Med 2018;379:1007-16.

42. U.S. Food and Drug Administration. FDA Drug Innovation > Novel Drug Approvals for 2018 [Internet]. FDA Drug Innovation > Novel Drug Approvals for 2018, 2019. Available: https://www.fda.gov/Drugs/DevelopmentApprovalProcess/Druglnnovation/ ucm592464.htm [Accessed 30 Jan 2019]

43. Nishimura RA, Otto CM, Bonow RO, et al. 2017 AHA/ACC Focused Update of the 2014 AHA/ACC Guideline for the Management of Patients With Valvular Heart Disease: A Report of the American College of Cardiology/American Heart Association Task Force on Clinical Practice Guidelines. J Am Coll Cardiol 2017;70:252-89.
44. Baumgartner H, Falk V, Bax JJ, et al. 2017 ESC/EACTS guidelines for the management of valvular heart disease. Eur Heart J 2017;38:2739-91.

45. Sannino A, Smith RL, Schiattarella GG, et al. Survival and cardiovascular outcomes of patients with secondary mitral regurgitation: a systematic review and meta-analysis. JAMA Cardiol 2017;2:1130-9.

46. Obadia J-F, Messika-Zeitoun D, Leurent $G$, et al. Percutaneous repair or medical treatment for secondary mitral regurgitation. N Engl J Med 2018;379:2297-306.

47. Mack MJ, Abraham WT, Lindenfeld J, et al. Cardiovascular outcomes assessment of the MitraClip in patients with heart failure and secondary mitral regurgitation: design and rationale of the COAPT trial. Am Heart J 2018;205:1-11.

48. Nishimura RA, Bonow RO. Percutaneous repair of secondary mitral regurgitation - a tale of two trials. N Engl J Med 2018;379:2374-6.

49. Kirklin JK, Pagani FD, Kormos RL, et al. Eighth annual INTERMACS report: Special focus on framing the impact of adverse events. J Heart Lung Transplant 2017;36:1080-6.

50. Patel CB, Cowger JA, Zuckermann A. A contemporary review of mechanical circulatory support. J Heart Lung Transplant 2014;33:667-74.

51. Mehra MR, Naka Y, Uriel N, et al. A fully magnetically levitated circulatory pump for advanced heart failure. N Eng/ J Med 2017;376:440-50.

52. Mehra MR, Goldstein DJ, Uriel N, et al. Two-year outcomes with a magnetically Levitated cardiac pump in heart failure. N Engl J Med 2018;378:1386-95.

53. Mehra MR, Salerno C, Cleveland JC, et al. Healthcare resource use and cost implications in the momentum 3 long-term outcome study. Circulation 2018:138:1923-34.

54. Mehra MR, Salerno C, Naka Y, et al. A tale of the twist in the outflow graft: an analysis from the momentum 3 trial. J Heart Lung Transplant 2018;37:1281-4.

55. Wang TJ, Larson MG, Levy $D$, et al. Temporal relations of atrial fibrillation and congestive heart failure and their joint influence on mortality: the Framingham Heart study. Circulation 2003;107:2920-5.

56. Kuck K-H, Brugada J, Fürnkranz A, et al. Cryoballoon or radiofrequency ablation for paroxysmal atrial fibrillation. N Engl J Med 2016;374:2235-45.

57. Cosedis Nielsen J, Johannessen $A$, Raatikainen $P$, et al. Radiofrequency ablation as initial therapy in paroxysmal atrial fibrillation. N Engl J Med 2012:367:1587-95.

58. Marrouche NF, Brachmann J, Andresen $D$, et al. Catheter ablation for atrial fibrillation with heart failure. N Engl J Med 2018;378:417-27.

59. Poole JE. CABANA Trial - Recurrence of Atrial Arrhythmias in the Catheter Ablation Versus Antiarrhythmic Drug Therapy for Atrial Fibrillation [Internet]. Clinical Trials Update presented at: ESC Congress 2018. Munich, Germany, 2018.

60. Solomon SD, Zelenkofske S, McMurray JJV, et al. Sudden death in patients with myocardial infarction and left ventricular dysfunction, heart failure, or both. $N \mathrm{Eng} / \mathrm{J}$ Med 2005;352:2581-8.

61. Moss AJ, Zareba W, Hall WJ, et al. Prophylactic implantation of a defibrillator in patients with myocardial infarction and reduced ejection fraction. N Engl J Med 2002:346:877-83.

62. Moss AJ, Hall WJ, Cannom DS, et al. Improved survival with an implanted defibrillator in patients with coronary disease at high risk for ventricular arrhythmia. Multicenter Automatic Defibrillator Implantation Trial Investigators. N Engl J Med 1996;335:1933-40.

63. Buxton $A E$, Lee $K L$, Fisher JD, et al. A randomized study of the prevention of sudden death in patients with coronary artery disease. Multicenter Unsustained Tachycardia Trial Investigators. N Engl J Med 1999;341:1882-90.

64. Hohnloser SH, Kuck KH, Dorian P, et al. Prophylactic use of an implantable cardioverter-defibrillator after acute myocardial infarction. $N$ Eng/ J Med 2004;351:2481-8

65. Steinbeck G, Andresen D, Seidl K, et al. Defibrillator implantation early after myocardial infarction. N Engl J Med 2009;361:1427-36.

66. Olgin JE, Pletcher MJ, Vittinghoff E, et al. Wearable cardioverter-defibrillator after myocardial infarction. N Engl J Med 2018;379:1205-15.

67. Vaduganathan $M$, Solomon SD. Expanding the global borders of heart failure: the shop and people studies. Eur Heart J 2018;39:1781-3.

68. McMurray JJV, Krum H, Abraham WT, et al. Aliskiren, enalapril, or aliskiren and enalapril in heart failure. N Engl J Med 2016;374:1521-32. 